Conclusions: Epigenetic cell counting is a promising novel tool to reproducibly and easily quantify immune cells in the (inflamed) labial salivary gland of sicca patients with relatively low amount of tissue needed $\left(<1 \mathrm{~mm}^{3}\right)$. Considering the potential of this technique to include a huge number of (cell-specific) biomarkers we believe this opens up new standardized ways for salivary gland analysis with high relevance for patient classification, understanding of immunopathology and clinical trials.

Disclosure of Interest: None declared

DOI: 10.1136/annrheumdis-2017-eular.4933

\section{THU0235 EXOSOME-DELIVERED MIR-146A REGULATES SENESCENCE OF BONE MARROW- MESENCHYMAL STEM CELLS FROM SYSTEMIC LUPUS ERYTHEMATOSUS PATIENTS THROUGH TARGETING IRAK1 AND TRAF6}

J. Ji, Y. Wu, X. Kong, Z. Zhang, Z. Gu ${ }^{1,1}$. Department of Rheumatology, Affiliated Hospital of Nantong University, Nantong, China

Background: Exosomes are membrane nano-vesicles secreted by a multitude of cells that harbor biological constituents such as proteins, lipids, mRNA and microRNA. Recent study suggests that microRNAs can be transferred between cells and mediate target gene repression. Our research group revealed the senescence of bone marrow-mesenchymal stem cells from systemic lupus erythematosus patients, which participated in the development of SLE. However, the relationship between senescence of MSCs and miRNAs remains unclear. Objectives: In this study, we investigated whether exosomes act as intercellular messengers delivering microRNA that modulate the senescence of BM-MSCs from SLE patients and its possible mechanism.

Methods: Tewelve female SLE patients and healthy subjects were enrolled in the study. All patients were females, and their age distribution was similar to that of the cases. Serum were collected from these persons. All BM-MSCs were Isolated by density gradient centrifugation. Serum-derived exosomes were extracted by Total Exosome Isolation reagent and confirmed by transmission electron microscope and western blot. The internalization of exosomes was detected by immunofluorescence. QRT-PCR was used to distinguish the difference of expression of miR-146a in exosomes between normal group and SLE group. Different exosomes stimulated normal BM-MSCs, then detecting expression of miR146a by qRT-PCR, detecting expression of IRAK1 and TRAF6 by WB, observing the activity of $\beta$-gal of cells, the changes of cytoskeletal structure by F-actin staining and the distribution of cell cycle by flow cytometry. We used miRNA mimics and miRNA inhibitor to interfere the expression of miR-146a.

Results: Serum-derived exosomes could be taken up by BM-MSCs through the plasma membrane due to treatment of BM-MSCs with exosomes. After stimulation of exosomes in normal MSCs, miR146a was decreased, but, IRAK1 and TRAF6 was activated. And, the cell volume and the number of SA- $\beta$-gal positive in SLE BM-MSCs was increased. The organization of cytoskeleton was neatly disordered. The rate of cell proliferation was decreased. The miR-146a mimics in SLE BM-MSCs can significantly reverse the senescence.

Conclusions: Exosomes-delivered miR-146a in the serum of SLE patients can promote the senescence of BM-MSCs through targeting IRAK1 and TRAF6. Exosomes play an important role in the pathogenesis of SLE.

Acknowledgements: This research was supported by grants from the National Natural Science Foundation of China (81471603)

Disclosure of Interest: None declared

DOI: 10.1136/annrheumdis-2017-eular.6033

\section{THU0236 ANTIBODIES TOWARDS ATP-BINDING CASSETTE TRANSPORTER ABCA1: A NEW MECHANISM FOR ATHEROSCLEROSIS IN SLE?}

M. Fernandes Das Neves ${ }^{1,2}$, J.R. Batuca ${ }^{3}$, F. Batista ${ }^{2}$, C. Favas ${ }^{2}$, H. Célia ${ }^{2}$, J. Delgado Alves ${ }^{2} \cdot{ }^{1}$ CEDOC - Chronic Disease Research Center, NOVA Medical School/Faculdade de Ciências Médicas, Universidade Nova de Lisboa, Lisbon;

${ }_{2}^{2}$ Medicine IV, Professor Doutor Fernando Fonseca Hospital, Amadora; ${ }^{3}$ CEDOC

- Chronic Disease Research Center, NOVA Medical School/Faculdade de

Ciências Médicas, Universidade Nova de Lisboa, Lisbon, Portugal

Background: Systemic Lupus Erythematosus (SLE) is considered an independent risk factor for cardiovascular disease and patients with SLE have an increased burden of atherosclerotic vascular disease ${ }^{1}$. High-density lipoproteins (HDL) are the plasma lipoproteins responsible for reverse cholesterol transport ${ }^{2}$. HDL protective effect on cardiovascular disease is attributed to the cholesterol efflux capacity as well as to its anti-oxidant and anti-inflammatory properties ${ }^{3}$. Dyslipidemia is frequent amongst patients with SLE, characteristically with low HDL levels.

ATP-binding cassette transporter ABCA1, also known as the cholesterol efflux regulator protein, is a ubiquitous cholesterol transporter that is highly expressed in macrophages. Its main function is to donate cholesterol to apolipoprotein A-I (ApoA-I) in lipid-poor HDL particles. As such, ABCA1 closely influences HDL levels and its role in atherosclerosis has been increasingly studied ${ }^{4}$.

Objectives: This study was undertaken to determine if antibodies against ABCA1 can be detected in patients with SLE through enzyme-linked immunosorbent assay (ELISA).
Methods: Patients with SLE were divided in two groups: group A, with low damage (based on less than 4 SLICC criteria), and group B, with high damage (based on the presence of at least 4 SLICC criteria). Groups A and B were compared with a control group. 48 patients were enrolled (13 in group $A$ and 35 in group B), and 18 age and gender-matched healthy controls were included in the control group. IgG anti-ABCA1 and anti-HDL antibodies were assessed by home-made ELISAs, using commercially available ABCA1 synthetic peptide and HDL from healthy donors.

Results: There were no differences between group $A$ and the control group. Group B had higher titers of anti-ABCA1 antibodies when compared with group $A(p=0.016)$ and the control group $(p=0.022)$. For positivity we considered values superior to 3 standard deviations above the mean of healthy controls. Four patients showed positive anti-ABCA1 titers (11.4\%).

Conclusions: This is the first time that naturally occurring antibodies against ABCA1 are detected by ELISA. These antibodies are increased in patients with SLE that have higher damage, measured by SLICC classification criteria. Future studies will determine their pathogenic role and the potential use of a standardized ELISA to detect anti-ABCA1 antibodies in clinical practice.

References:

[1] Stojan G, Petri M. Atherosclerosis in systemic lupus erythematosus. J Cardiovasc Pharmacol. 2013; 62(3):255-262.

[2] Lewis GF, Rader DJ. New insights into the regulation of HDL metabolism and reverse cholesterol transport. Circ Res. 2005;96:1221-1232.

[3] Navab M, Yu R, Gharavi N, et al. High-density lipoprotein: antioxidant and anti-inflammatory properties. Curr Atheroscler Rep. 2007;9(3):244-8.

[4] Soumian S, Albrecht C, Davies AH, Gibbs RGJ. ABCA1 and atherosclerosis. Vasc Med. 2005; 10:109-119.

Disclosure of Interest: None declared

DOI: 10.1136/annrheumdis-2017-eular.7013

\section{THU0237 WHOLE TRANSCRIPTOME ANALYSIS OF APL TREATED HUVECS MAPS PROINFLAMMATORY AND PROCOAGULANT PATHWAYS}

M.D. Patsouras ${ }^{1}$, S. Foutadakis ${ }^{2}$, E. Alexopoulou ${ }^{2}$, M. Agelopoulos ${ }^{2}$, D. Thanos ${ }^{2}$, A.G. Tzioufas ${ }^{1}$, P.G. Vlachoyiannopoulos ${ }^{1} .{ }^{1}$ Department of Pathophysiology, Medical School National Kapodistrian University; ${ }^{2}$ Biomedical Research Foundation Academy, Athens, Greece

Background: Antiphospholipid syndrome is an autoimmune thrombophillia characterized by recurrent thromboembolism and or pregrancy morbidity in the presence of antiphospholipid antibodies (aPL). $\beta 2 \mathrm{GPI}$ which is the major autoantigen in the syndrome forms complexes with anti-B2GPI autoantibodies that activate platelets, monocytes and endothelial cells. Previous studies have shown that anti- $\beta 2$ GPI- $\beta 2$ GPI complexes activate TLR4 and TLR6 on endothelial cells leading to $F_{K} B$, MAPK activation and Tissue Factor and proinflammatory cytokine expression. ${ }^{1,2}$

Objectives: To evaluate the whole transcriptome of endothelial cells that have been stimulated with aPL-B2GPI complexes.

Methods: Human umbilical Vein Endothelial cells (HUVECs) were isolated from 2 APS patients and 4 Healthy control women upon delivery. Healthy donor HUVEC were stimulated with IgG isolated from APS patients with high aPL titers and healthy individuals in the presence of B2GPI. Consequently total mRNA was isolated, cDNA libraries were created and whole transcriptome sequencing (RNASeq) was performed. Gene expression data were validated in protein levels with immunohistochemistry in placenta tissues from APS patients and healthy individuals.

Results: Whole transcriptome analysis of HUVECs stimulated with APL- $\beta 2$ GPI complexes and IgG from healthy individuals revealed 680 differentially expressed genes, among which 377 were upregulated and 303 downregulated in the aPL stimulated endothelial cells. Characteristic examples of the upregulated genes are IL-6, IL-8, VCAM1, SELE and TGFB2 and TGFBR1. Bioinformatics analysis revealed that the upregulated genes belong mainly to the cytokinecytokine receptor interaction (hsa053323), MAPK signaling pathway (hsa04010), TNF signaling pathway (hsa04668) and NOD-like receptor pathway (hsa04621). Characteristic examples of the downregulated genes include the CBX4, CBX8, $\mathrm{BCOR}$ and $\mathrm{HDAC7}$ genes. Interestingly some of the proteins encoded by these genes play role in the epigenetic modification of DNA. Immunohistochemical staining on placenta biopsies from APS patients and healthy individuals for IL-6, IL-8, IL-18, NFkB, TF, TNF-a, E-SELECTIN, MAPK8, TGFB2 and TGFBR1 showed increased intensity in the signal of endothelial cells on APS specimens validating thus the RNASeq results in the tissues.

Conclusions: RNASeq of endothelial cells treated with aPL and B2GPI reveals a thoroughly analysed proinflamatory and procoagulant phenotype. Moreover differential expression of DNA modifying proteins suggests the possible epigenetic regulation of gene expression on endothelial cells in APS syndrome. Ongoing experiments aim to analyze histone acetylation and methylation status of the promoters of the selected genes that were shown to be differentially expressed. References:

[1] Pierangeli SS, Vega-Ostertag ME, Gonzalez EB. New targeted therapies for treatment of thrombosis in antiphospholipid syndrome. Expert reviews in molecular medicine. 2007;9(30):1-15.

[2] Vega-Ostertag M, Casper K, Swerlick R, Ferrara D, Harris EN, Pierangeli 